\title{
Control of Q-factor in nanobeam cavities on substrate
}

\author{
Danilo Panettieri ${ }^{1}$, Liam O'Faolain ${ }^{2}$, Marco Grande ${ }^{1, *}$ \\ ${ }^{I}$ Dipartimento di Ingegneria Elettrica e dell'Informazione (DEI), Politecnico di Bari, Italy \\ ${ }^{2}$ SUPA, School of Physics and Astronomy, University of St. Andrews, UK
}

\begin{abstract}
In this paper, we demonstrate how to efficiently control the quality factor of silicon nitride nanobeam cavities, grown on a silica substrate and embedded in an upper cladding, by engineering the nanobeam cross-section and the shape of the periodic holes. We propose optimized configurations that are able to overcome the decreasing of the Q-factor when the nanobeam is embedded in an asymmetric medium. More precisely, we show that the maximum achievable quality factor can be designed and tuned in asymmetric configurations where the upper cladding is particularly different from the substrate one. These optimized configurations exhibit high-Q factor and small mode volume over a wide range of the upper cladding refractive index paving the way for the realization of innovative optical sensors and for the compensation of fabrication tolerances in embedded optical nanobeam cavities.
\end{abstract}

Keywords: nanobeams, cavities, asymmetric structures, low contrast waveguides, sensing.

\section{INTRODUCTION}

Optical cavities showing high quality factors are essential building blocks for many applications such as nonlinear optics [1], refractive index sensing [2], enhanced emission [3] and filters [4] to mention a few. In twodimensional photonic crystals (2D-PhCs), light confinement is achieved by taking advantage of both photonic bandgaps (in the plane) and total internal reflection (perpendicular direction), respectively. PhC nanocavities are formed by the addition of defects in the periodic lattice, and indeed the optimization of the in-plane and out-ofplane losses of the cavity is strictly necessary to achieve high-Q factors and low mode volumes.

However, when dealing with low refractive index contrast waveguides, two-dimensional photonic crystals lack the possibility to achieve high Q-factors. This behavior is worsened if asymmetric configurations are taken into account, i.e. when the nanobeam is supported and embedded in two different dielectric media [5].

In recent years, a valid alternative to planar $2 \mathrm{D}-\mathrm{PhCs}$ was theoretically and experimentally proposed by the introduction of optical one-dimensional (1D) nanobeams that can show very high Q-factors in the order of $10^{9}$ when the nanobeam is air suspended [6]. Moreover, nanobeams offer large photonic band-gaps, even when the index contrast between the period structure and the background is low, and permit to attain small mode volumes by applying specific design to the structure.

Nanobeam cavities are widely used in refractive index sensing devices [7]. More often, these devices require disconnected periodic features that imply the introduction of a supporting substrate. At the same time, asymmetric nanobeams can be found in integrated optical devices such as vertical couplers [4]. Therefore, the refractive index difference between the upper and bottom cladding degrades the performance of the optical cavity because of the coupling between the transverse electric (TE) and transverse magnetic (TM) modes [8]. For this reason, a homogeneous "encapsulation" is the better solution for attaining high Q-factor.

In this work, we consider a silicon nitride $\left(\mathrm{Si}_{3} \mathrm{~N}_{4}\right)$ nanobeam on a silica $\left(\mathrm{SiO}_{2}\right)$ substrate and embedded in a dielectric medium. Silicon nitride is an optimal choice for the realization of optical cavities operating in the visible and near-infrared ranges due to its relatively large refractive index, its transparency and its biocompatibility. Up to now, $\mathrm{Si}_{3} \mathrm{~N}_{4}$-based nanobeams have been proposed in several applications ranging from optomechanics [9], to quantum dot [10] to optical biosensing [11]. In these applications, this choice is also motivated by the necessity to have a structure with strong mechanical and thermal stability and that could also be CMOS compatible.

Initially, we design and optimize a $\mathrm{Si}_{3} \mathrm{~N}_{4}$ nanobeam embedded in homogeneous material $\left(\mathrm{SiO}_{2}\right)$ to obtain high Qfactor and then we investigate the effects of the upper cladding refractive index variation onto the nanobeam performance. Finally, we show how to reduce the coupling between the modes by engineering the cross section of the waveguide nanobeam and the shape of the holes constituting the taper and the mirror. The final designs allow achieving the maximum Q-factor for asymmetric configurations even when the upper refractive index is particularly different from the substrate counterpart.

\section{NANOBEAM CAVITY DESIGN}

Figure 1(a) depicts the geometry of the $\mathrm{Si}_{3} \mathrm{~N}_{4}$ nanobeam cavity consisting of a waveguide perforated with periodic holes (the hole shape is sketched in the inset of Figure 1(a)), separated by a constant distance, and a 
defect localized in between the two mirrors. Furthermore, tapered holes are added inside the cavity in order to reduce the modal mismatch between the cavity and the mirrors.

Due to the low refractive index contrast, it is necessary to carefully optimize the design to achieve high quality factors. In this regard, we exploit elliptical gratings instead of circular holes since elliptical gratings offer larger bandgap and higher reflectivity leading to a better confinement of the optical mode [7].

The nanobeam thickness $h$ and width $w$ are equal to $400 \mathrm{~nm}$ and $600 \mathrm{~nm}$, respectively. The periodicity is set equal to $310 \mathrm{~nm}$ in order to set the cavity resonance wavelength at about $1000 \mathrm{~nm}$.

To obtain the tapering, a quadratic increment of the aspect ratio of the hole dimensions $\left(l_{z} / l_{x}\right)$ is employed. In particular, the major diameter of the elliptical hole $\left(l_{z}\right)$, is incremented between $l_{z \min }=200 \mathrm{~nm}$ and $l_{z \max }=400 \mathrm{~nm}$, while the minor diameter of the ellipse $\left(l_{x}\right)$, is unaffected. In a similar fashion, the quadratic law is applied to the distance between the holes starting by the cavity, between $a_{\min }=285 \mathrm{~nm}$ and $a=310 \mathrm{~nm}$. Finally, we set the number of taper holes $T$ and the number of mirror holes $M$ equal to 10 and 40, respectively, to obtain high Qfactor.
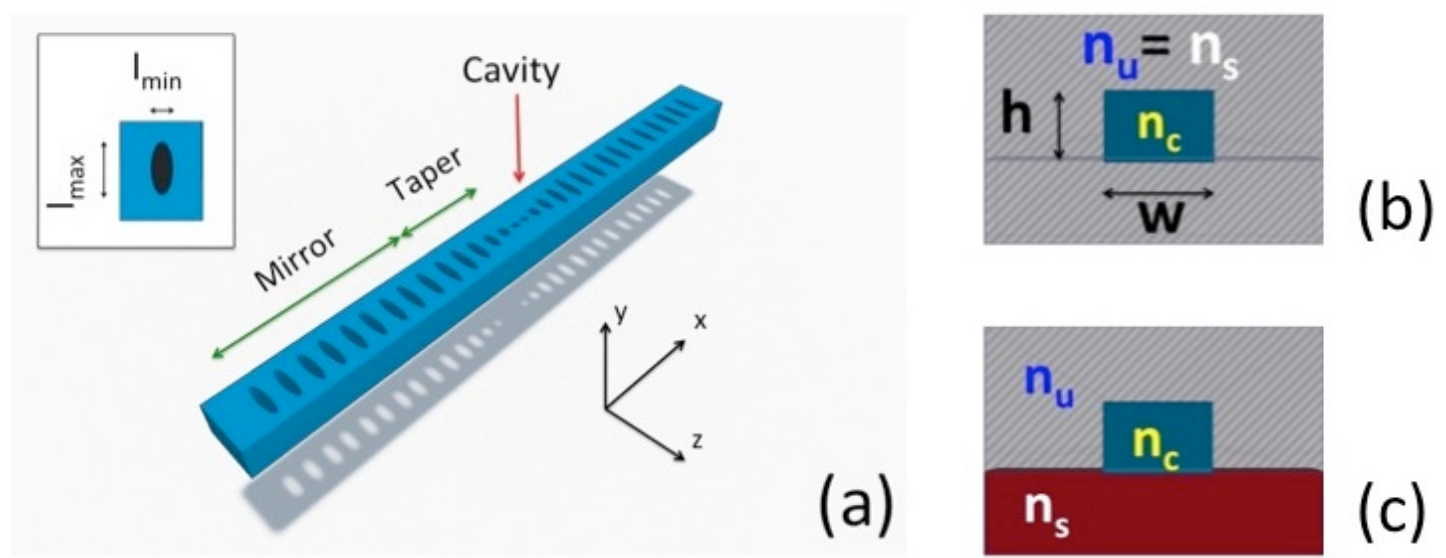

Figure 1. (a) Sketch of the $\mathrm{Si}_{3} \mathrm{~N}_{4}$ nanobeam consisting of two mirrors and two tapered regions; (inset) geometrical parameters of the periodic hole. Cross-section of the (b) symmetric and (c) asymmetric configurations, respectively where $n_{c}, n_{s}$ and $n_{u}$ are the core, substrate and upper cladding refractive indexes.

The structure shown in Figure 1(a) is initially completely embedded in $\mathrm{SiO}_{2}$ with all holes infiltrated as shown in Figure 1(b). In the following numerical analysis, the refractive index of the nanobeam $n_{c}$ is set equal to 2 while the refractive index of the background and the upper cladding is equal to $n_{s}=n_{u}=1.5\left(\mathrm{SiO}_{2}\right)$. The numerical analysis was performed by means of the FDTD method using the RSoft suite [13].

We started our analysis by determining the optimum cavity length $L_{c}$ equal to $82 \mathrm{~nm}$ (the cavity length is defined as the distance between the inner holes of the taper) that leads to an operating wavelength close to $1000 \mathrm{~nm}$. The optimized design offers a Q-factor in the order of $10^{5}$ as shown in Figure $2\left(n_{u}=1.5\right)$. However, when the structure becomes asymmetric (i.e. $n_{u} \neq n_{u}$ ), the Q-factor degrades rapidly because of the loss due to coupling through the transverse magnetic TM and transverse electric TE modes [8]. In particular, Figure 2 shows a rapid decrease of the Q-factor (about one order of magnitude) when the refractive index difference is about 0.1 . We also verified that this behavior is independent with respect to the cavity length. Therefore, these findings clearly highlight a limit of nanobeam cavities when the upper cladding material does not exactly match the substrate counterpart. 


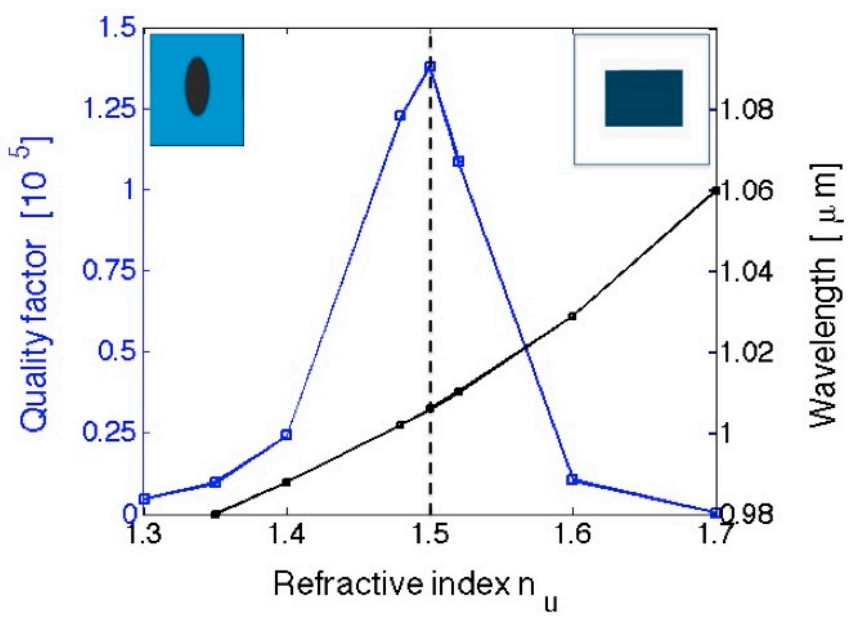

Figure 2. $Q$-factor versus the refractive index of the upper cladding (blue y-axis) when the substrate refractive index $n_{s}$ is equal to 1.5. The dashed black line refers to the symmetric configuration; (inset) cross-section of the $\mathrm{Si}_{3} \mathrm{~N}_{4}$ nanobeam. (Secondary y-axis, in black) Resonant wavelength of the nanobeam cavity when the cavity length $L_{c}$ is equal to $82 \mathrm{~nm}$.

\section{OPTIMIZATION OF THE ASYMMETRIC CONFIGURATION}

In many applications, the substrate and the upper cladding have different refractive indexes and, usually, the nanobeam is supported by a fixed substrate (e.g. $\mathrm{SiO}_{2}$ ) and embedded in a lower refractive index as in sensing devices or integrated vertical couplers.

In this scenario, it is possible to modify the nanobeam cross-section in order to alter the cavity behavior. In particular, we consider a nanobeam whose thickness $h$ equal to $300 \mathrm{~nm}$ while its width $w$ is extended to $750 \mathrm{~nm}$. Moreover, the elliptical hole size $l_{z}$ is slightly modified between $l_{z \max }=500 \mathrm{~nm}$ and $l_{z \min }=200 \mathrm{~nm}$ with a quadratic law.

Figure 3(a) shows the Q-factor when the upper cladding is varied. The plot clearly reveals that the maximum achievable Q-factor is obtained for an asymmetric configuration $\left(n_{s}=1.5, n_{u}=1.45\right)$. This behavior can further emphasized by modifying the hole shape and introducing elongated holes. As it can be inferred by the inspection of Figure 3(b), the maximum achievable Q-factor shifts to $n_{u}=1.4$ when the substrate refractive index $n_{s}$ is kept constant and equal to 1.5. Furthermore, it is worth comparing the Q-factor when $n_{u}=1.35$ since the configuration with elongated hole-based configuration shows a Q-factor 5 times higher than the first reported configuration (Figure 2) at the same value of $n_{u}$. Finally, these configurations maintain a very high Q-factor over a wide range of upper cladding refractive indexes, revealing a strong fabrication tolerance, and a reduced dependency on surrounding medium.

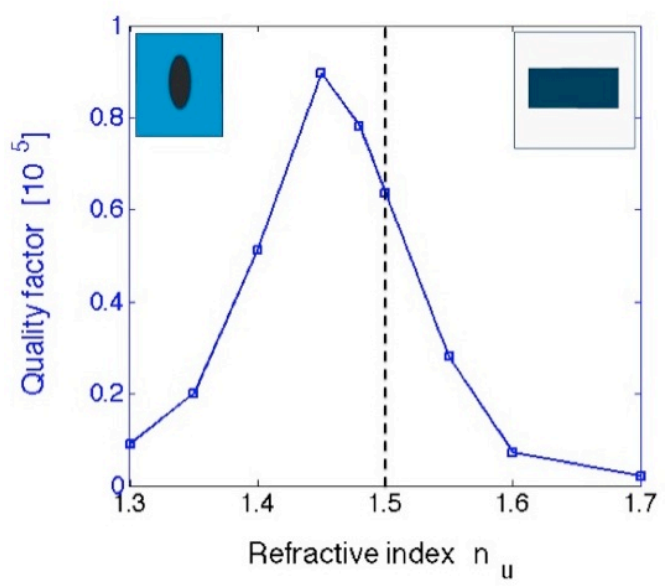

(a)

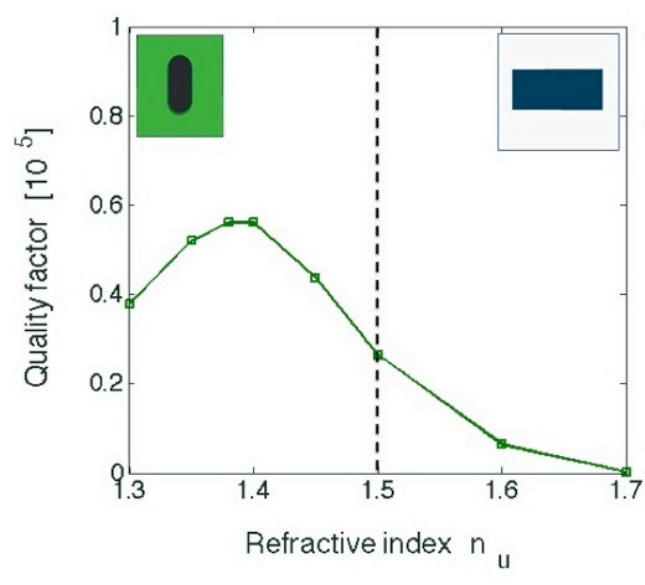

(b)

Figure 3. $Q$-factor versus the refractive index of the upper cladding when the substrate refractive index $n_{s}$ is equal to 1.5 for the (a) elliptical hole and (b) elongated hole, respectively. The dashed black lines refer to the symmetric configuration; (inset) cross-section of the $\mathrm{Si}_{3} \mathrm{~N}_{4}$ nanobeam. The cavity length $L_{c}$ is equal to $76 \mathrm{~nm}$.

Table I summarizes the geometrical parameters of the optimized configurations. 
Table I. Geometrical parameters of the optimized nanobeam cavities.

\begin{tabular}{|l|c|c|c|}
\hline & Elliptical hole [1] & Elliptical hole [2] & Elongated hole \\
\hline Cross section $[h \times w]$ & $400 \times 600$ & $300 \times 750$ & $300 \times 750$ \\
\hline Hole aspect-ratio $\left[l_{z} / l_{x}\right][\mathrm{nm}]$ & $200 / 400$ & $200 / 500$ & $200 / 500$ \\
\hline Cavity length $L_{c}[\mathrm{~nm}]$ & 82 & 76 & 76 \\
\hline Maximum Q-factor & 134790 & 89626 & 56078 \\
\hline Shift maximum Q-factor & $0 \%$ & $10 \%$ & $20 \%$ \\
\hline
\end{tabular}

\section{CONCLUSIONS}

In conclusion, we report on different geometrical configurations for the optimization of the nanobeam cavity Qfactor when the nanobeam is embedded in a low refractive index. In particular, we show how to control the Qfactor over a wide range of upper cladding refractive indexes when the cross-section and the hole shape is fully engineered. This approach provides a $5 x$ increase in the achievable Q-factor and a shift of about $20 \%$ for the asymmetric configuration with upper and lower cladding materials.

Therefore, the presented configurations could be exploited for the realization of innovative optical sensors. Furthermore, these configurations will foster the realization of embedded optical cavities (e.g. vertical couplers) with negligible fabrication tolerance since the high Q-factor is almost constant over a very large refractive index range.

\section{ACKNOWLEDGEMENTS}

D. Panettieri thanks the Politecnico di Bari for "Best Erasmus traineeship for jobs" grant. L. O'Faolain acknowledges support from a European Research Council Starting grant (no. 337508). M. Grande thanks the Apulia Region program "FutureInResearch" (7K76VI3) for financial support.

\section{REFERENCES}

[1] S. Buckley, M. Radulaski, J. L. Zhang, J. Petykiewicz, K. Biermann, and J. Vučković, "Multimode nanobeam cavities for nonlinear optics: high quality resonances separated by an octave," Opt. Express, vol. 22, no. 22, pp. 26498-509, 2014

[2] M. G. A. Rahman, P. Velha, R. M. De La Rue, and N. P. Johnson, "Silicon-on-insulator (SOI) nanobeam optical cavities for refractive index based sensing," Spie, vol. 8439, no. Figure 1, p. 84391Q, 2012.

[3] R. L. Savio, S. L. Portalupi, D. Gerace, A. Shakoor, T. F. Krauss, L. O'Faolain, L. C. Andreani \& M. Galli "Room-temperature emission at telecom wavelengths from silicon photonic crystal nanocavities," Applied Physics Letters 98, 201106 (2011).

[4] Grande, M., L. O’Faolain, T. P. White, M. Spurny, A. D’Orazio, and T. Krauss, "Optical filter with very large stopband $(\approx 300 \mathrm{~nm})$ based on a photonic crystal vertical directional coupler," Optics Letters, vol. 34, No. 21, 3292-3294, 2009.

[5] B.-S. Song, S.-W. Jeon, and S. Noda, "Symmetrically glass-clad photonic crystal nanocavities with ultrahigh quality factors," Opt. Lett., vol. 36, no. 1, pp. 91-3, 2011.

[6] Q. Quan, and M. Loncar, "Deterministic design of high Q, small mode volume photonic crystal nanobeam cavities," Opt. Express 19, 18529-18542 (2011).

[7] Q. Quan, I. B. Burgess, S. K. Y. Tang, D. L. Floyd, and M. Loncar, "High-Q, low index-contrast polymeric photonic crystal nanobeam cavities," Opt. Express, vol. 19, no. 22, pp. 22191-22197, 2011.

[8] Y. Tanaka, T. Asano, R. Hatsuta, and S. Noda, "Investigation of point-defect cavity formed in twodimensional photonic crystal slab with one-sided dielectric cladding," Appl. Phys. Lett., vol. 88, no. 1, pp. 7-10, 2006.

[9] M. Davanço, S. Ates, Y. Liu, and K. Srinivasan, " $\mathrm{Si}_{3} \mathrm{~N}_{4}$ optomechanical crystals in the resolved-sideband regime," Appl. Phys. Lett., vol. 104, no. 4, 2014.

[10] C. Lee, T. Frost, W. Guo, and P. Bhattacharya, "Integration of 1.3- $\mu \mathrm{m}$ quantum-dot lasers with $\mathrm{Si}_{3} \mathrm{~N}_{4}$ Waveguides for Single Mode Optical Interconnects," IEEE Journal of Quantum Electronics, vol. 48, no. 10, pp. 1346-1351, 2012.

[11] P. Muellner, E. Melnik, G. Koppitsch, J. Kraft, F. Schrank, and R. Hainberger, "CMOS-compatible $\mathrm{Si}_{3} \mathrm{~N}_{4}$ waveguides for optical biosensing," Procedia Eng., vol. 120, pp. 578-581, 2015.

[12] RSoft Suite, FullWAVE user guide. 